Revue d'histoire de l'Amérique française

DEVUE D.HISTOIRE DE L'AMÉRIQUE FRANÇAISE

\title{
Note de recherche : À la recherche des Deux Montagnes
}

\section{Jean-Paul Ladouceur}

Volume 52, numéro 3, hiver 1999

URI : https://id.erudit.org/iderudit/005498ar

DOI : https://doi.org/10.7202/005498ar

Aller au sommaire du numéro

Éditeur(s)

Institut d'histoire de l'Amérique française

ISSN

0035-2357 (imprimé)

1492-1383 (numérique)

Découvrir la revue

Citer cet article

Ladouceur, J.-P. (1999). Note de recherche : À la recherche des Deux Montagnes. Revue d'histoire de l'Amérique française, 52(3), 383-406. https://doi.org/10.7202/005498ar

\section{Résumé de l'article}

Cette recherche avait pour but de trouver l'origine du toponyme Deux Montagnes qui désigne ce grand lac au sud-ouest de Montréal, et de localiser ces deux montagnes à l'origine du nom. Au cours des années et pour des raisons diverses, la situation de ces deux entités géographiques s'est perdue, en sorte que l'on propose aujourd'hui plusieurs endroits différents pour ces deux collines ou montagnes dont on peut penser qu'elles sont connues depuis des millénaires. 


\title{
A LA RECHERCHE DES DEUX MONTAGNES
}

\author{
JEAN-PAUL LADOUCEUR
}

\section{RÉSUMÉ}

Cette recherche avait pour but de trouver l'origine du toponyme Deux Montagnes qui désigne ce grand lac au sud-ouest de Montréal, et de localiser ces deux montagnes à l'origine du nom. Au cours des années et pour des raisons diverses, la situation de ces deux entités géographiques s'est perdue, en sorte que l'on propose aujourd'hui plusieurs endroits différents pour ces deux collines ou montagnes dont on peut penser qu'elles sont connues depuis des millénaires.

\section{ABSTRACT}

The objective of this research was to find the origin of the toponym Deux Montagnes that refers to the large V-shaped lake in the South-West of Montreal, and to locate the two mountains from which the name originates. For various reasons, the position of these two geographical entities was lost over the years. Today, many locations for the two hills or mountains, which have perhaps been known for millenia, are proposed.

Trouver l'origine d'un nom de lieu est souvent difficile et parfois impossible, surtout lorsque son usage remonte à quelques centaines d'années et que les documents attestant de son utilisation sont rares ou inexistants. C'est le cas du toponyme Deux Montagnes qui désigne ce grand lac en forme de V évasé au sudouest de l'île de Montréal. On ignore depuis quand ce toponyme est en usage et où sont situées les deux montagnes à l'origine du nom.

On ne peut que spéculer sur le début de l'usage de cette appellation car, avant 1674 , les documents anciens, cartes ou récits de voyageurs sont muets sur ce point. Quant à l'emplacement des deux montagnes, les personnes, les organismes et les documents consultés ont indiqué au moins six endroits différents pour ces deux entités et ce n'est qu'après avoir refait le parcours des premiers voyageurs dans des conditions semblables à celles qui étaient les leurs qu'il fut possible d'éliminer les localisations qui semblaient peu vraisemblables et de proposer celle qui convenait le mieux à ce point de repère remarquable que constituaient ces deux montagnes sur la route de l'Ouest. 


\section{L'ORIGINE DU TOPONYME}

L'utilisation attestée du toponyme Deux Montagnes pour désigner le lac remonte à $1674^{1}$, mais on ignore s'il doit son origine à l'une ou l'autre des tribus indiennes qui parcouraient la région avant l'arrivée des Européens ou bien s'il est le fait des explorateurs et voyageurs français ou canadiens qui, très tôt au XVII siècle, naviguèrent sur cette nappe d'eau. On sait toutefois que la partie nordouest du lac et la rivière des Outaouais furent pendant longtemps la principale voie de pénétration à l'intérieur du continent et, de ce fait, très fréquentée.

Dans sa relation de 1635, le père Jean de Brébeuf rapporte que le voyage de Québec jusqu'au lac Huron est «[...] très fâcheux, non seulement à raison de sa longueur et de la mauvaise chère qu'on y fait, mais encore pour les circuits qu'il faut faire de Québec jusques ici par les Bissiriniens et la Petite Nation. Je crois qu'il y en a pour plus de trois cents lieues. Il est vrai que le chemin est plus court par le Sault-de-Saint-Louis et par le lac des Iroquois [le lac Ontario], mais la crainte des ennemis et le peu de commodités qui s'y rencontrent en rend [sic] le passage désert ${ }^{2}$.»

Peu après avoir quitté Sainte-Anne-de-Bellevue, les voyageurs entraient dans le lac des Deux Montagnes et apercevaient droit devant eux, non loin de l'actuel village d'Oka, la pointe aux Bleuets dominée à l'arrière par deux montagnes. Cette pointe dont le nom est d'usage récent (après 1900) fut, dans le passé, un endroit stratégique important, car de ce point, on pouvait contrôler tout le trafic de la rivière des Outaouais et celui qui pouvait survenir de la partie est du lac. Des fouilles archéologiques ont d'ailleurs montré la présence sporadique d'Indiens à cet endroit depuis la période archaïque ${ }^{3}$, soit plusieurs centaines d'années avant l'arrivée des Blancs. Comme les Indiens utilisaient des noms descriptifs pour désigner les lieux, on pourrait penser que le toponyme Deux Montagnes a pu désigner cette pointe, puis le lac et être ensuite traduit en français par les Blancs. Or il n'existe aucune preuve ni aucun indice qui permettent d'avancer que le lac ou cette pointe avait un nom avant l'arrivée des Blancs, ou que le toponyme Deux Montagnes soit une traduction d'un nom indien.

Les Indiens considéraient peut-être qu'il ne s'agissait pas d'un lac, mais plutôt de l'élargissement de deux rivières (rivière des Outaouais et des Mille Îles). Sur les cartes topographiques actuelles, on indique encore entre parenthèses Rivière des Outaouais sous l'inscription Lac des Deux Montagnes, dans la partie ouest du lac. Il est aussi possible qu'à une certaine époque, les deux montagnes n'aient été qu'un point de repère sur la route de l'Ouest. L'origine du toponyme demeure mystérieuse, car les documents disponibles ne permettent pas de désigner qui, des Blancs ou des Indiens, l'aurait ainsi nommé.

1. Gouvernement du Québec, Commission de toponymie, Noms et lieux du Québec (SainteFoy, 1994), 176 [à l'avenir: Noms et lieux du Québec].

2. Jean de Brébeuf, Écrits en huronie (Montréal, Bibliothèque québécoise, 1996), 9.

3. Sylvie Lalonde, Le patrimoine historique de la région d'Oka (Ministère du Loisir, de la Chasse et de la Pêche, Rapport non publié, octobre 1985), 13. 
Au tout début de la colonie, Champlain nomma le lac de deux noms différents, soit: lac de Médicis sur sa carte de 1612 et lac de Soissons sur celle de 1632. La première appellation était en l'honneur de Marie de Médicis et la deuxième voulait célébrer la mémoire de Charles de Bourbon, comte de Soissons ${ }^{4}$. Si le lac avait eu un nom, Champlain l'aurait peut-être utilisé sur ses cartes.

Certains citoyens de la région et d'ailleurs 5 prétendent que le toponyme Deux Montagnes a été donné par Champlain et en veulent pour preuve les écrits de ce dernier. Cette thèse n'a aucun fondement, car on ne trouve nulle part dans les écrits de Champlain le nomde Deux Montagnes pour désigner le lac, même s'il a écrit qu'il est venu sur cette nappe d'eau en 1611 «[...], je fis quelques [sic] huit lieues par terre cottoyant le grand saut par des bois qui sont assez clairs, et fus jusques à un lac, $\left.[. . .]^{6}\right\rangle$. Ce qui a peut-être induit ces personnes à penser ainsi est la note infrapaginale que ceux qui ont réédité les écrits de Champlain ont ajoutée et qui indique que le lac en question était le lac des Deux Montagnes?.

Le nomactuel de l'établissement amérindien est Kanesatake, nom d'origine iroquoise qui «[...] signifierait "au bas de la côte" [...] proviendrait plutôt de l'ancien site qu'occupaient antérieurement les Mohawks sur l'île de Montréal, au pied du mont Royal $^{\natural}$ ». Daprès un autre auteur ${ }^{9}$, Kanesatake serait un mot agnier signifiant «sur les dunes» et aurait été en usage avant le toponyme Oka.

Dans son Lexique de la langue iroquoise ${ }^{10}$, le linguiste J. A Cuoq traduit ainsi Kanesatake: «[...] de onesa, pente, déclin, côte, et de ehtake, au bas, en bas. Litt. au bas de la côte. Le village du Lac des deux Montagnes est en effet situé au pied d'une colline.» Par ailleurs, dans son Lexique de la langue algonquine ${ }^{11}$, le même auteur indique à l'article Kanactage: «c'est le mot iroquois Kanesatake qui signifie "au bas de la montagne". Ce mot employé par les Iroquois de la mission du Lac des Deux-Montagnes a passé dans la langue algonquine comme nom propre de lieu et signifie Lac des Deux-Montagnes.» Enfin, dans un mémoire soumis à la Société royale du Canada en 1893 et intitulé Anotc kekon ${ }^{12}$ qui signifie mélanges, ce même auteur utilise la forme Kanactageng (le Lac ou au Lac) pour désigner le même lieu. Le nomactuel correspond donc à celui du premier établissement iroquois au pied du mont Royal.

4. Noms et lieux du Québec, 176.

5. J. Urgel Demers, Esquisse monographique de la paroisse du patronage de Saint-Josephdu-Lac (Montréal, 1960), 9.

6. Euvres de Champlain présentées par Georges-Émile Giguère (Montréal, Éditions du Jour, 1973), 1: 242 .

7. On trouve une note semblable dans les Écrits de Champlain présentés par C.-H. Laverdière à la fin du siècle dernier.

8. Noms et lieux du Québec, 307.

9. Lorraine Létourneau, «Oka», Okami. Journal de la société d'histoire d'Oka, 11,3 (septembre 1987): 15 .

10. J. A. Cuoq, Lexique de la langue iroquoise avec notes et appendices (Montréal, J. Chapleau et Fils, [1883]), 10.

11. J. A. Cuoq, Lexique de la langue algonquine (Montréal, J. Chapleau et Fils, 1886), 144.

12. J. A. Cuoq, Anotc kekon. Mémoires de la Société royale du Canada, Section I, Tome XI, $1893,150$. 


\section{L'ANCIENNETÉ ET L'ÉCRITURE DUTOPONYME}

Les documents anciens fournissent des renseignements sur la date à laquelle le toponyme a été en usage, les différentes façons dont on l'a écrit, de même que d'autres indications qui permettent de privilégier une localisation plutôt qu'une autre pour les deux montagnes.

Dans le dictionnaire des noms de lieux du Québec ${ }^{13}$, on indique que le plus vieux document cartographique sur lequel on a trouvé l'appellation Deux Montagnes pour désigner le lac est une carte de Jean-Baptiste Franquelin en date de 1684. Toutefois, le même dictionnaire signale un peu plus loin que le nom devait être utilisé $<[\ldots]$ avant cette date par la population, car le père jésuite Antoine Delmas y fait allusion en 1674 dans le compte rendu de son voyage d'exploration autour de lîle de Montréal ${ }^{14} \gg$. Sur les cartes et plans rédigés sous le Régime français, le nomest généralement orthographié Lac des Deux Montagnes, même si on trouve parfois la forme abrégée Lac des 2 montagnes ${ }^{15}$ et même L des 2 Mont $^{16}$.

Sous le Régime anglais, les noms de lieux, génériques et spécifiques, sont, à peu d'exceptions près, traduits en anglais et l'appellation devient Lake of the Two Mountains ou Lake of Two Mountains. Jusqu'à la fin des années 1950, sur la plupart des cartes publiées par le gouvernement fédéral, c'est la forme anglaise qui a prévalu. Sur les cartes topographiques on traduisait même le nom du comté «county of Two Mountains ${ }^{17}{ }^{7}$. Â partir de 1960, le nomaété retraduit en français sur pratiquement toutes les cartes publiées par ce même gouvernement. Exceptionnellement, d'autres noms ont aussi été utilisés pour désigner le lac, dont lac Maupas ${ }^{18}$ et lac de la Chaudière ${ }^{19}$.

\section{LASITUATIONDES DEUX MONTAGNES}

Le toponyme Deux Montagnes étant un nom descriptif, on pourrait s'attendre à ce que la localisation de ces deux montagnes ne pose pas de difficulté dans un endroit comme la plaine de Montréal; mais c'est loin d'être le cas. En effet, on dénombre non pas deux mais plusieurs collines ${ }^{20}$ ou montagnes dans la région d'Oka (figure 1), sans

13. Noms et lieux du Québec, 176.

14. Ibid.

15. V. de Belmont, Carte du cours du Saint-Laurent depuis son embouchure jusques et y compris le lac Supérieur (Ottawa, Archives publiques du Canada (APC), National Map Collection [NMC], [1680].

16. Jean-Baptiste Bourguignon d'Anville, [Carte de 1'] Amérique Septentrionale (Paris, Ottawa, APC, NMC, 1746).

17. Gouvernement du Canada, Department of National Defense, Army Survey Establishment, Lachine, 31H5, West Half (Fourth Edition, 1:50000, 1952).

18. Noms et lieux du Québec, 177.

19. Guillaume de L'Isle, Carte du Canada qui comprend la partie septentrionale des Etats Unis d'Amérique (Paris, chez Dezanche, 1783), NMC.

20. Les gens de la région d'Oka appellent montagnes la douzaine de modestes élévations qui parsèment leur coin de pays. Le terme colline serait beaucoup plus approprié pour les désigner, car la plus haute ne dépasse pas 270 mètres ( 886 pieds) et plusieurs ont à peine 100 mètres ( 328 pieds). Tout au long du présent texte, les génériques colline et montagne sont utilisés indifféremment. 
compter la montagne de Rigaud au sud-ouest du lac. De plus, les ouvrages et les personnes consultés indiquent plusieurs endroits différents pour ces deux montagnes à l'origine du nomdu lac.

Certains ${ }^{21}$ pensent que les deux montagnes sont deux ensembles de collines, soit celles d'Oka et celles de Saint-André2. Pour sa part, la Commission de toponymie du Québec propose deux endroits diffé rents: «Au moins deux hypothèses existent au sujet de l'emplacement des deux montagnes qui ont servi à la création de ce toponyme descriptif. Certains les placent sur la rive nord du lac, évoquant ainsi probablement les deux plus hauts sommets des collines d'Oka, soit le mont Bleu et le Calvaire. Dautres voient dans l'ensemble des collines d'Oka et la montagne de Rigaud, situées de part et d'autre du lac, la raison de cette appellation ${ }^{23}$.»

La tradition populaire nous apprend que les deux montagnes sont le $\mathrm{Cal}-$ vaire à Oka et la montagne de Rigaud ${ }^{24}$, mais deux auteurs ${ }^{25}$ de la région affirment que ce sont le Calvaire et le mont Saint-Sulpice. Enfin, un géographe important a remarqué que: «Les collines de l'ouest [Covey Hill, Rigaud et Oka] sont au nombre de trois; nous pourrions dire quatre, étant donné que celle d'Oka comporte deux masses jumelles baptisées les Deux-Montagnes ${ }^{26} . \gg$ Les deux masses jumelles sont la montagne du Calvaire et cette autre montagne à l'est de la mine désaffectée entre la route 344, l'autoroute 640 et la montée du Village. En dernier lieu, l'auteur d'un ouvrage sur la ville de DeuxMontagnes ${ }^{27}$ présente la version du Conseil de la culture des Laurentides et celle de la Commission de toponymie du Québec mais ne prend parti ni pour l'une ni pour l'autre.

Finalement, sur la plupart des cartes anciennes sur lesquelles deux montagnes sont représentées au moyen de symboles ${ }^{28}$, ces derniers sont situés au nord-est d'Oka ou du «village indien» et désignent la montagne du Calvaire et

21. Conseil de la culture des Laurentides, Le chemin des seigneuries (s.l., n.é., n.d.), 15.

22. Les collines de Saint-André sont plus petites que celles d'Oka: la plus élevée a 131 mètres (430 pieds) et deux autres dépassent à peine 100 mètres.

23. Noms et lieux du Québec, 176.

24. Dans une brochure rédigée à l'occasion du $150^{\mathrm{e}}$ anniversaire de la municipalité de SainteScholastique, le curé Georges Duquet écrit: «Le lac en effet s'écoule entre deux montagnes. Par contre, la topographie [...] présente plusieurs montagnes formant une chaîne. Soit qu'on considère cette chaîne comme une montagne ou comme plus que deux; dans ce dernier cas, le nom devient une mauvaise désignation; dans le premier cas, il faut se rendre à l'évidence que l'autre montagne est sur l'autre versant du lac à Rigaud.», Sainte-Scholastique, 1825-1975 (s.l., n.é., n.d.), 5.

25. André De Pagès, «Appellation du lac des Deux-Montagnes», Okami. Journal de la société d'histoire d'Oka, 7,2 (1992): 23 et Stella Dupuis-Mailhot, Le lac des Deux-Montagnes (Société d'histoire d'Oka inc., 1992), 1.

26. Raoul Blanchard, L'ouest du Canada français. Montréal et sa région (Montréal, Beauchemin, 1953), 1: 22.

27. Stéphane Plante, Ville de Deux-Montagnes, 1804-1994 (Deux-Montagnes, 1993), 17-18.

28. Toutes les cartes anciennes consultées ont été publiées avant 1816 et, sur 14 de ces dernières (voir la liste en annexe), les deux montagnes sont représentées au moyen de symboles. 


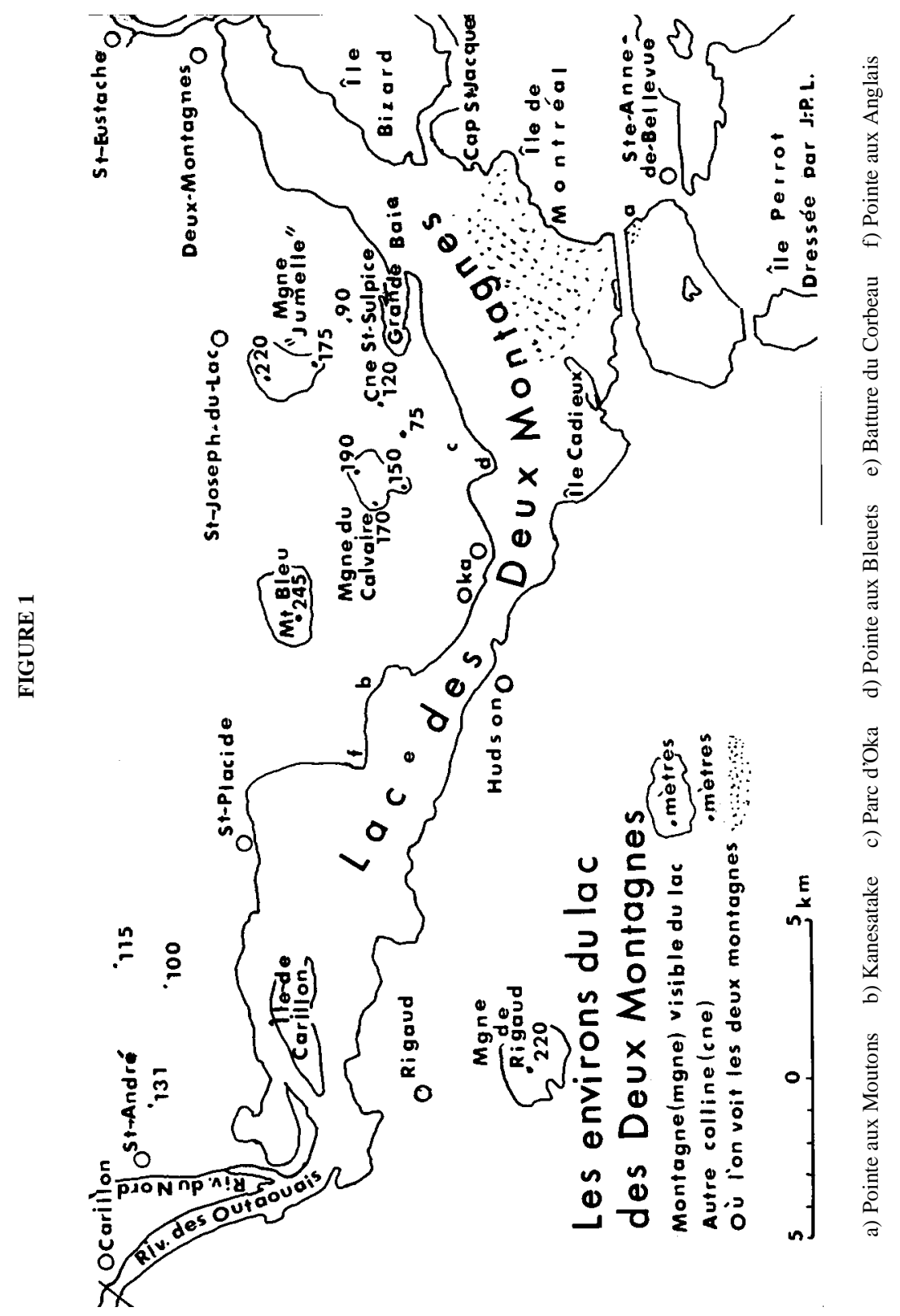


sa jumelle au nord-est (figure 2). Toutefois, sur deux de ces cartes ${ }^{29}$, l'un des symboles est disposé près d'Oka et l'autre à l'endroit du mont Bleư ${ }^{30}$

\section{LA DIFFICILE LOCALISATION}

Il n'est pas facile d'expliquer pourquoi autant de lieux sont proposés pour ces deux montagnes, mais l'une des premières raisons est peut-être que l'on a oublié que, à l'origine, le nom fut donné par des voyageurs indiens, français ou canadiens qui se déplaçaient en canot sur le lac et non à pied sur la terre ferme. La vision qu'ils avaient du paysage était très différente de celle que les habitants du lieu ont pu avoir par la suite en s'installant dans la région ou en la traversant par les chemins, alors que les arbres cachaient tout ou partie de ce même paysage.

\section{SUR LE LAC}

Pour voir ce que les Indiens et les voyageurs ont vu et avoir une chance de localiser ce point de repère remarquable que constituaient les deux montagnes sur la route des Pays-d'en-Haut, il est essentiel de refaire, à bord d'une embarcation, le même parcours qu'eux..

Outre son étendue considérable, ce qui frappe d'abord l'observateur est la forme en $\mathrm{V}$ évasé de ce lac. La branche nord-ouest qui s'étend de l'embouchure de la rivière du Nord à la pointe ouest de l'île de Montréal a plus de trente kilomètres et sa branche nord-est en compte environ dix-huit ${ }^{31}$. Il ne faut pas non plus s'attendre à voir des montagnes jeunes avec des sommets pointus, car le relief des collines d'Oka, tout comme celui de la montagne de Rigaud, est de même nature que celui des Laurentides et, comme ce dernier, il est le résultat de l'action érosive des glaciers qui ont arrondi toutes les formes.

C'est la branche nord-ouest du lac, dans laquelle plusieurs n'ont vu qu'un prolongement ou un élargissement de la rivière des Outaouais qui était empruntée par les voyageurs. À partir de Montréal, ces derniers traversaient le lac SaintLouis et s'arrêtaient après un premier portage à Sainte-Anne-de-Bellevue. Ils profitaient de cet arrêt pour demander la protection de la sainte et poursuivaient ensuite à travers la branche nord-ouest du lac, la rivière des Outaouais jusqu'à la rivière Mattawa et, par cette dernière, jusqu'à la baie Géorgienne et le lac Huron.

29. Samuel Gale et John B. Duberger, Plan of Part of the Province of Lower Canada [...], 1795 et Samuel Holland, A New Map of the Province of Lower Canada (London, Faden, 1813).

30. Ce toponyme (mont Bleu) est en usage localement, mais ne semble pas encore très bien établi. Sur les cartes topographiques on le désigne par «mont Saint-Alexis». Les répertoires toponymiques publiés par la Commission de toponymie du Québec en 1969, 1971 et 1978 indiquent SaintAlexis, tandis que Noms et lieux du Québec, publié en 1994 par ce même organisme, le désigne par l'appellation mont Bleu (p. 176). Dans la région d'Oka, la plupart des personnes interrogées ne connaissent pas de mont Saint-Alexis.

31. On situe habituellement la limite entre le lac et la rivière des Mille Îles au pont de chemin de fer qui relie la municipalité de Deux-Montagnes à l'île Jésus. 
FIGURE 2

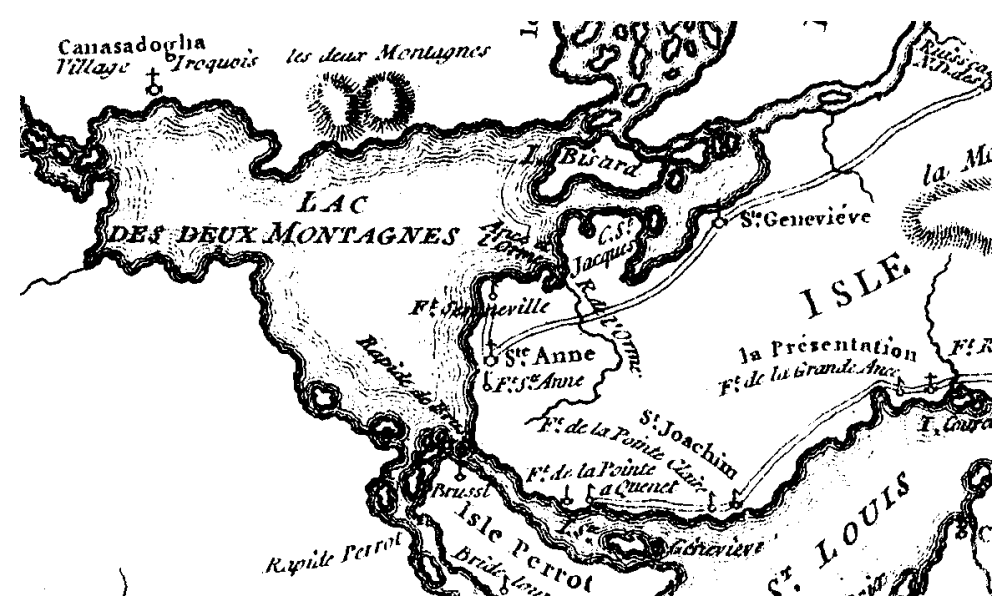

Partie de la carte A New Map of the Province of Québec According to the Royal Proclamation [...], 1776, Captain Carver and other officers.

Cette partie du lac était un passage obligé et elle fut pendant longtemps très fréquentée dans un sens comme dans l'autre.

\section{LA TRAVERSÉE DE LA BRANCHE NORD-OUEST DU LAC}

Peu après avoir quitté Sainte-Anne-de-Bellevue, soit aux environs de la pointe aux Moutons, à l'extrémité ouest de l'île de Montréal, les voyageurs apercevaient à l'horizon (figure 3), droit devant eux, sur la rive nord du lac, deux formes arrondies, assez semblables, séparées par un espace plus ou moins plat. Vus du lac, ces deux formes ou arcs de cercle presque plats paraissent n'être formés chacun que d'une seule colline, à cause de la couleur verte uniforme de la végétation mais, en fait, deux sommets forment la première colline et deux, la deuxième. Dans l'espace plat entre les deux arcs, on distingue une petite élévation du côté gauche, c'est le monticule que l'on a nommé le mont Saint-Sulpice. La montagne du Calvaire au centre du premier arc n'a que 152 mètres de hauteur, mais derrière, au nord-est, se profile un autre sommet de 190 mètres. Le tout forme un seul arc de cercle, une seule colline.

La deuxième forme arrondie, la montagne «Jumelle ${ }^{32} »$, se trouve à l'est et au nord de l'ancienne mine de niobium entre le rang Sainte-Sophie, l'autoroute 640 et la route 344 (chemin d'Oka). Au nord du point de rencontre de la route

32. Au long du texte, la première forme arrondie, le premier arc est désigné par la montagne du Calvaire et la deuxième forme par la montagne «Jumelle». 


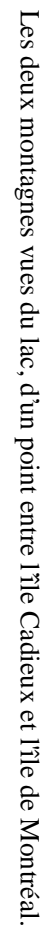

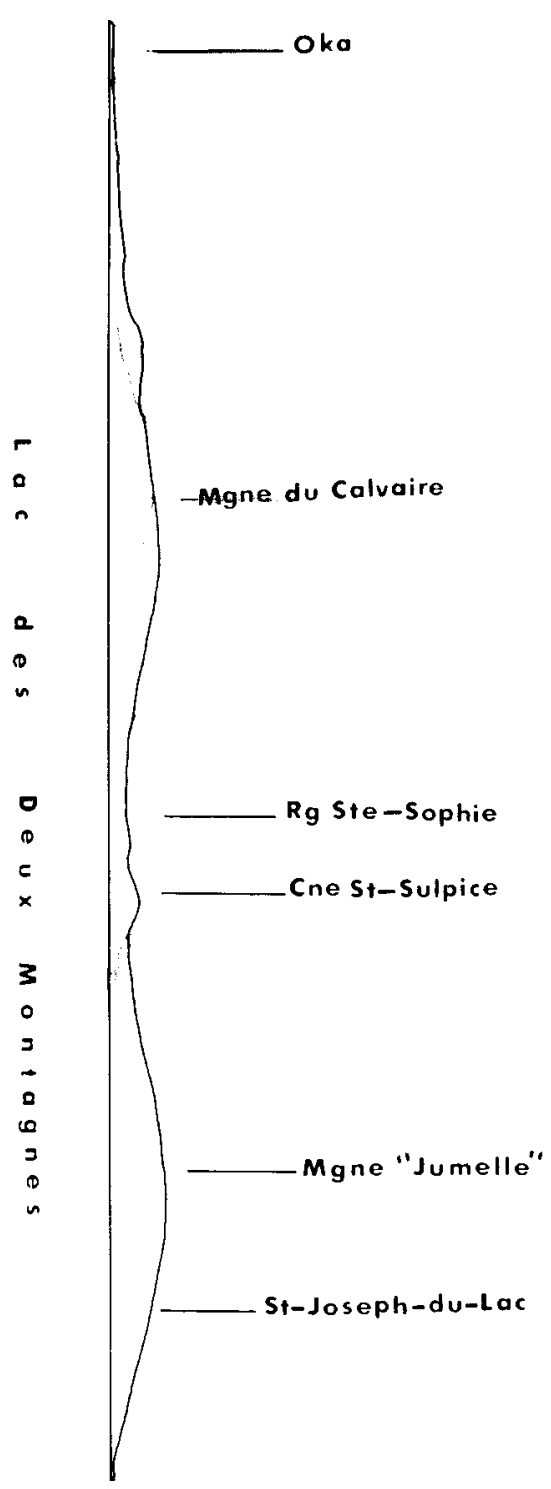

匀 
344 avec le rang Saint-Isidore, près de la limite est de la municipalité de paroisse d'Oka, la pente est très raide, la colline s'élève rapidement jusqu'à 170 mètres pour redescendre un peu par la suite et remonter jusqu'à 220 mètres non loin de la courbe de la montée du Village ${ }^{33}$. Sur le flanc nord de cette élévation s'est établi le village de Saint-Joseph-du-Lac. C'est pourquoi on appelle parfois le plus haut sommet, montagne de Saint-Joseph, mais cette dernière appellation n'est pas utilisée pour désigner la première colline, celle de 170 mètres.

Sur l'eau, le pont de l'île aux Tourtes (autoroute 40) empêche de bien voir ces deux collines, mais aussitôt de l'autre côté de cet ouvrage, elles sont parfaitement visibles. De ce point, ces deux formes, à peu près égales, ne paraissent pas très élevées, mais plus on s'avance dans le lac, plus elles prennent de l'importance et se détachent nettement sur la ligne d'horizon. Ce sont les deux seules collines ou montagnes qui sont visibles et elles ont ceci de particulier que ce sont deux masses qui se côtoient et qui sont de forme, de hauteur et d'importance à peu près égales. Ces caractéristiques ne peuvent être observées que du lac ou du bout de lîle de Montréal, dans cette partie de Senneville qui fait face au parc d'Oka. Sur la terre ferme du côté nord du lac, on ne peut voir en même temps les deux montagnes par manque du recul nécessaire.

En continuant sur le lac, ces deux collines se détachent nettement à l'horizon jusqu'aux environs de l'île Cadieux: plus loin vers l'ouest, la perspective change, la montagne du Calvaire devient plus importante que sa jumelle au nord-est, puis elle disparaît complètement à son tour passé le village d'Oka.

On peut aussi observer ces deux collines sur une partie de la branche nordest du lac entre l'île Cadieux et le cap Saint-Jacques; plus loin au nord, la perspective change, les trois ou quatre bosses de faible envergure en arrière de la Grande Baie, mais en avant des deux montagnes, cachent le $U$ de la vallée et on ne distingue plus les deux montagnes, car la ligne d'horizon n'est affectée que d'une légère courbe entre les deux élévations. Plus loin encore, la montagne «Jumelle» cache complètement la montagne du Calvaire et ce phénomène est peut-être à l'origine du dicton populaire qui dit qu'il y a bien deux montagnes, mais que l'une cache l'autre.

Après le village d'Oka, la falaise et les arbres qui la surmontent sur la rive nord cachent toutes les collines qui se trouvent derrière et ce n'est qu'après avoir dépassé le village d'Hudson, à mi-chemin entre cette dernière localité et la pointe aux Anglais que l'on aperçoit le mont Bleu au nord. À l'ouest se profile une masse aplatie qui dépasse la cime des arbres: la montagne de Rigaud. Plus on s'avance vers l'ouest, plus ces deux élévations deviennent des éléments importants du paysage. Le mont Bleu se détache de l'ensemble des collines d'Oka et se

33. La $7^{\mathrm{e}}$ édition de la carte topographique Lachute, 31G9, 1984 (1:50 000), comporte une erreur. Sur une courbe maîtresse de la montagne «Jumelle», on a indiqué 150 mètres au lieu de 200. Le sommet a donc 220 mètres de haut et non 170 mètres. Sur l'édition précédente ( $6^{\mathrm{e}}$ édition, 1976) de cette même feuille, les courbes de niveau étaient cotées en pieds et la plus haute courbe pour ce sommet était de 700 pieds. 
profile très nettement à l'horizon. De même, la montagne de Rigaud grossit et sa forme s'apparente à celle d'un trapèze vis-à-vis de la municipalité de Saint-Placide.

\section{LE RETOUR PAR LA BRANCHE NORD-OUEST}

Lorsque ces mêmes voyageurs, revenant des Pays-d'en-Haut par la rivière des Outaouais, arrivaient au pied du Long Saut, site actuel du barrage de Carillon, ils apercevaient au loin une élévation qui, peu à peu, grossissait jusqu'à occuper la majeure partie du paysage du côté sud: la montagne de Rigaud. Un peu plus loin, après la courbe de la rivière, à environ un kilomètre à l'est de l'embouchure de la rivière du Nord, une deuxième masse se profilait droit devant eux à l'horizon: le mont Bleu.

Plus on s'avance vers l'est, par le chenal au sud de l'île de Carillon, plus ces deux collines deviennent importantes. Elles ne sont pas jumelles comme celles d'Oka, mais situées l'une en face de l'autre, de part et d'autre du lac, et on peut les observer à l'aller comme au retour. Que l'on passe au nord ou au sud de l'île de Carillon, les collines de Saint-André, moins hautes que celles d'Oka sont cachées par les arbres et ne sont pas visibles.

En résumé, de Sainte-Anne-de-Bellevue à Carillon, quatre collines ou montagnes seulement sont visibles du lac, si l'on excepte les trois ou quatre bosses d'une centaine de mètres derrière la Grande Baie. Ce sont: la montagne du Calvaire, la montagne «Jumelle», le mont Bleu et la montagne de Rigaud. Les trois premières font partie des collines d'Oka, tandis que la montagne de Rigaud, sur la rive sud du lac fait face au mont Bleu. De ces quatre montagnes, deux sont à l'origine du nom du lac.

Il est aussi important de noter que, lorsqu'on voit la montagne du Calvaire (figure 4), on voit aussi la montagne «Jumelle» au nord-est, mais qu'on ne peut voir ni la montagne de Rigaud ni le mont Bleu, tous deux situés à plus de cinq kilomètres au nord-ouest. De plus, lorsque l'on voit le mont Bleu, on voit aussi très bien la montagne de Rigaud sur l'autre rive du lac, mais il n'est pas possible d'apercevoir la montagne du Calvaire ni sa «Jumelle» au nord-est. Enfin, du côté nord du lac, passé le village d'Oka et jusqu'à Hudson, aucune montagne n'est visible, la falaise et les arbres qui la surmontent cachent tout. Du côté sud, la montagne de Rigaud ne commence à être visible qu'après le village d'Hudson aux environs de la batture du Corbeau.

\section{LES CARTES ANCIENNES}

Certaines cartes anciennes sont intéressantes parce qu'elles représentent les deux montagnes au moyen de symboles ${ }^{34}$, lesquels nous indiquent la situation de

34. Au moins 14 de ces cartes peuvent être consultées à la Bibliothèque nationale du Québec (voir la liste en annexe). Cette liste n'est pas exhaustive, car elle ne compte que des cartes publiées pendant la période de 1744 à 1815 . 


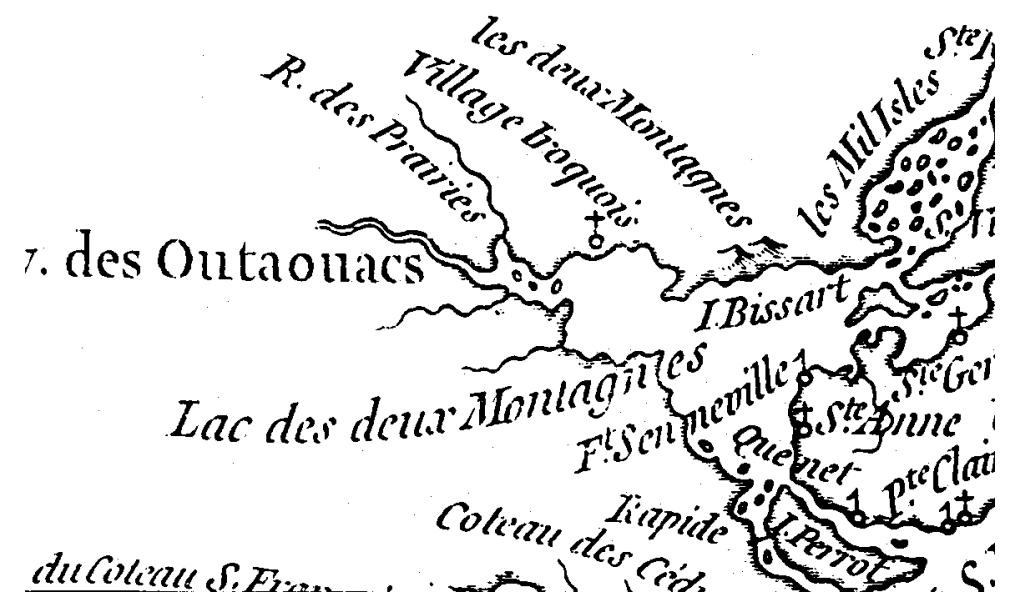

Partie de la carte Canada, Louisiane, terres anglaises [...], [d'Anville], 1755.

ces deux accidents géographiques. L'un des auteurs les plus prolifiques et l'un des premiers à ainsi représenter ces deux montagnes est Nicolas Bellin: six de ses cartes avec des symboles représentant les deux montagnes peuvent être consultées à la Bibliothèque nationale du Québec.

Les premières cartes de Bellin (cartes 1,2, 3, 4 de la liste) sont toutefois difficiles à interpréter. Les renseignements dont disposait l'auteur n'étaient pas très précis et le trait de côte est très généralisé et flou. Sur les deux premières cartes, il place les deux montagnes à l'ouest du lac, près de deux rivières (rivières Rouge et au Saumon), tandis que, sur les deux autres, les deux montagnes sont situées au milieu de la rive nord et, immédiatement à l'ouest de ces dernières, il situe «Le Long Sault».

Toutefois, dans son ouvrage intitulé Le petit atlas maritime: recueil de cartes et plans des quatre parties du monde publié en 1764, ce même auteur présente deux cartes (cartes 5 et 6 ) très intéressantes: la première (figure 5) décrit la Partie du fleuve Saint-Laurent depuis Québec jusqu'au lac Saint-François et la deuxième (figure 6) L'isle de Montréal et ses environs. Sur la première, la forme du lac a changé: il est maintenant en deux parties et les deux montagnes sont disposées sur la rive nord, au milieu de la partie nord-est du lac et vis-à-vis de l'entrée dans le lac par le passage entre l'île Perrot et l'île de Montréal. À l'extrémité de la partie ouest du lac est indiqué «Village d'Iroquois», de même qu'une rivière nommée «des Prairies» à l'endroit de l'actuelle rivière du Nord. Pour cette partie de la région, Bellin semble s'être très fortement inspiré d'une carte publiée 
en 1755 (carte 7) par le cartographe Jean-Baptiste Bourguignon d'Anville (figure 4): son trait de côte et sa toponymie sont pratiquement identiques.

Sa deuxième carte, L'isle de Montréal et ses environs (figure 6) a une échelle beaucoup plus grande que la précédente (2 lieues communes au lieu de 12) et son dessin est tout à fait remarquable, mais elle n'est pas exacte pour ce qui est du contour du lac. Ce dernier a une forme triangulaire et ne représente que la partie nord-est du lac, comme si l'auteur avait voulu indiquer que cette partie seulement du lac devait être considérée comme le lac des Deux Montagnes, la partie nordouest n'étant qu'un élargissement de la rivière des Outaouais. Les deux montagnes, très bien dessinées, occupent la rive nord-est du lac et font face au bout de l'île de Montréal et à l'entrée dans le lac par le passage entre l'île Perrot et l'île de Montréal. Ainsi situées, les deux montagnes ne peuvent qu'être la montagne du Calvaire et sa «Jumelle» au nord de la Grande Baie. Sur toutes les autres cartes à l'exception de deux, celle de Gale et Duberger en 1795 et celle de Holland en 1813 (cartes 12 et 13), les deux montagnes sont situées au même endroit, en face du passage plus haut mentionné.

Le mont Bleu est représenté au moyen d'un symbole pour la première fois en 1793, sur une carte de Jos. Peachy intitulée Plan of the River La Chinay [Lachenaie] or Milles Isles with the Lake of Two Mountains and Part of Ottawa River... (carte 10). Sur cette carte, la montagne du Calvaire est indiquée au nordest du village indien [Oka] et la montagne «Jumelle» au nord de la Grande Baie. Le mont Bleu, qui n'est pas identifié, est représenté plus loin au nord, à l'est de l'actuelle baie des Indiens. De toutes les cartes mentionnées dans la liste, c'est la seule qui identifie la montagne du Calvaire ${ }^{35}$. Sur la plupart des autres cartes, les symboles sont identifiés au moyen de l'inscription «les deux montagnes» ou «the two mountains». Sur les cartes de d'Anville, de Carver (figure 2) et celle du London Magazine (figure 7), cette désignation s'ajoute à celle du lac.

Sur les cartes de Gale et Duberger (figure 8) et de Holland ${ }^{36}$ deux montagnes seulement sont indiquées: la montagne du Calvaire au nord du village indien [Oka] et le mont Bleu au nord-est de l'actuelle baie des Indiens. Aucune des montagnes n'est identifiée sinon par l'inscription «Two Mountains» sur la carte de Holland. Il est difficile de croire que le nom du lac a pour origine la montagne du Calvaire et le mont Bleu comme semblent l'indiquer ces deux cartes, en raison de la distance entre ces deux montagnes et du fait que, du lac, lorsque l'on en voit une, l'autre n'est pas visible. Sur ces cartes, la montagne de Rigaud n'est pas indiquée. Pour ainsi situer les deux montagnes, on peut supposer que ces deux auteurs n'ont pas effectué leurs observations à partir d'une embarcation sur le lac.

La carte de Bouchette ${ }^{37}$ publiée au début du XIXe siècle (figure 9), après des levés de terrain et des enquêtes sur place, est la plus exacte et la plus précise: elle

35. La montagne est identifiée par l'inscription «The 7 chapels of mount Calv[aire].».

36. Voir la note 29.

37. Joseph Bouchette, Carte topographique de la province du Bas-Canada (Montréal, Éditions Elysée, 1980). Fac-similé de l'édition originale (London, Faden, 1815). 
FIGURE 5

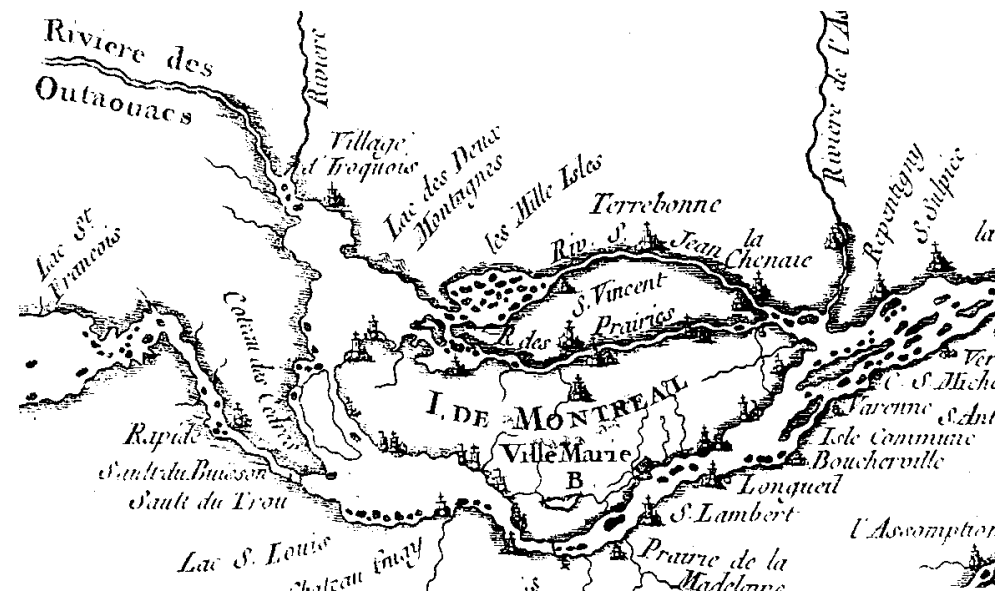

Partie de la carte Partie du fleuve Saint-Laurent depuis Québec jusqu'au lac Saint-François, 1764, [Bellin], Petit atlas maritime, carte 10.

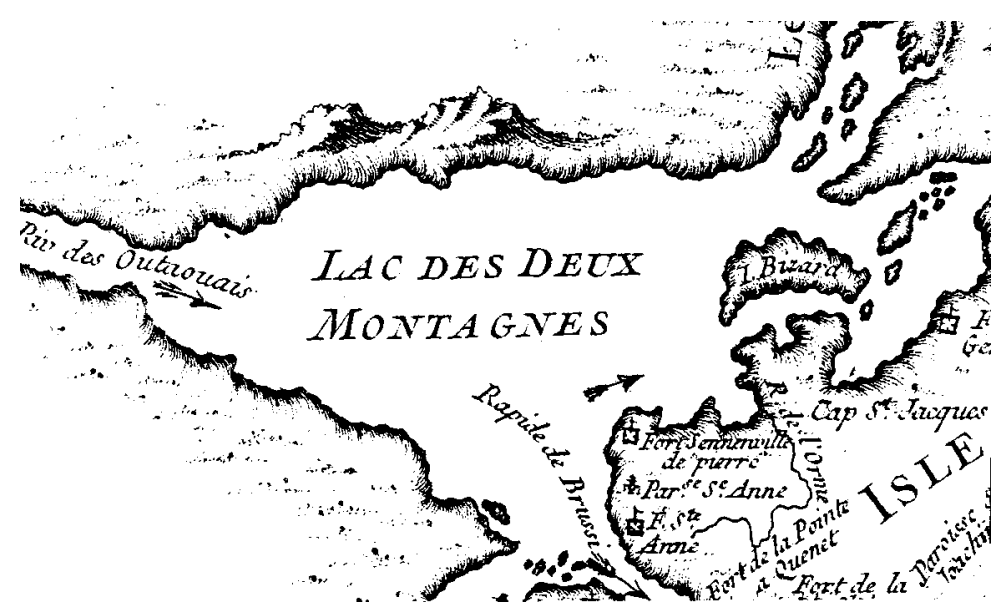

Partie de la carte L'Isle de Montréal et ses environs, 1764, [Bellin], Petit atlas maritime, carte 11. 
indique par des symboles très élaborés la montagne du Calvaire, la montagne «Jumelle» et la montagne de Rigaud, mais pas le mont Bleu (figure 9). Sur cette carte, aucune des montagnes n'est identifiée, mais Bouchette mentionne, dans son rapport, «le mont Calvart ${ }^{38} »$. Il s'agit évidemment de la montagne du Calvaire qui doit son nom aux sept petites chapelles construites sur son flanc est vers 1749 et qui contenaient chacune un bas-relief illustrant les souffrances du Christ sur le chemin de la Croix.

L'auteur a même indiqué sur sa carte le sentier qui mène aux trois chapelles du sommet et, sur la montagne «Jumelle», le chemin qui mène à Saint-Josephdu-Lac. Il n'a indiqué ni le mont Bleu ni les autres montagnes de la région au moyen de symboles, mais par un espace blanc parsemé de quelques arbres dans lequel il n'y a pas de champs cultivés.

\section{LES DIFFÉRENTES LOCALISATIONS}

À la suite des observations effectuées sur le lac et de l'étude des quelques cartes anciennes disponibles, il est maintenant possible d'évaluer les différentes localisations proposées pour les deux montagnes.

On ne peut considérer que l'une des deux montagnes soit «les collines de Saint-André» et l'autre «les collines d'Oka» comme on l'a curieusement proposé $^{39}$, car les collines de Saint-André ne sont pas visibles du lac, que l'on passe au sud ou au nord de l'île de Carillon.

La Commission de toponymie propose deux hypothèses pour les deux montagnes: le Calvaire et le mont Bleu ou bien l'ensemble des collines d'Oka et la montagne de Rigaud ${ }^{40}$. La première hypothèse est peut-être en partie basée sur la carte de Gale et Duberger et sur celle de Holland publiées à la fin de la période considérée, soit 1795 et 1813 . On notera aussi que les deux plus hauts sommets des collines d'Oka sont le mont Bleu (250 mètres) et la montagne «Jumelle» (220 mètres), la montagne du Calvaire n'a que 190 mètres. Cette hypothèse semble peu vraisemblable car, comme il a été observé, lorsque du lac on voit le mont Bleu, on voit aussi la montagne de Rigaud en face, de l'autre côté du lac, mais on ne peut absolument pas voir la montagne du Calvaire ni la montagne «Jumelle». De plus, au moins 9 autres cartes anciennes sur les 14 de la liste placent les deux montagnes au nord-est d'Oka et non au nord de la partie ouest du lac. Pour les mêmes raisons, la deuxième hypothèse (l'ensemble des collines d'Oka et la montagne de Rigaud) ne semble pas plus valable. L'ensemble des collines d'Oka n'est

38. «À peu de distance de l'Ottawa se trouvent les deux montagnes remarquables qui donnent le nom à la seigneurie et au lac; l'une d'elles nommée le Mont Calvart présente sur son sommet les restes de quelques bâtiments qui ont longtemps porté le nom des Sept-Chapelles.» Joseph Bouchette, Description topographique de la province du Bas-Canada avec des remarques sur le Haut-Canada et sur les relations des deux provinces avec les États-Unis de l'Amérique (Londres, 1815) (Montréal, Éditions Elysée, 1978), 106.

39. Voir la note 21 .

40. Noms et lieux du Québec, 176. 
FIGURE 7

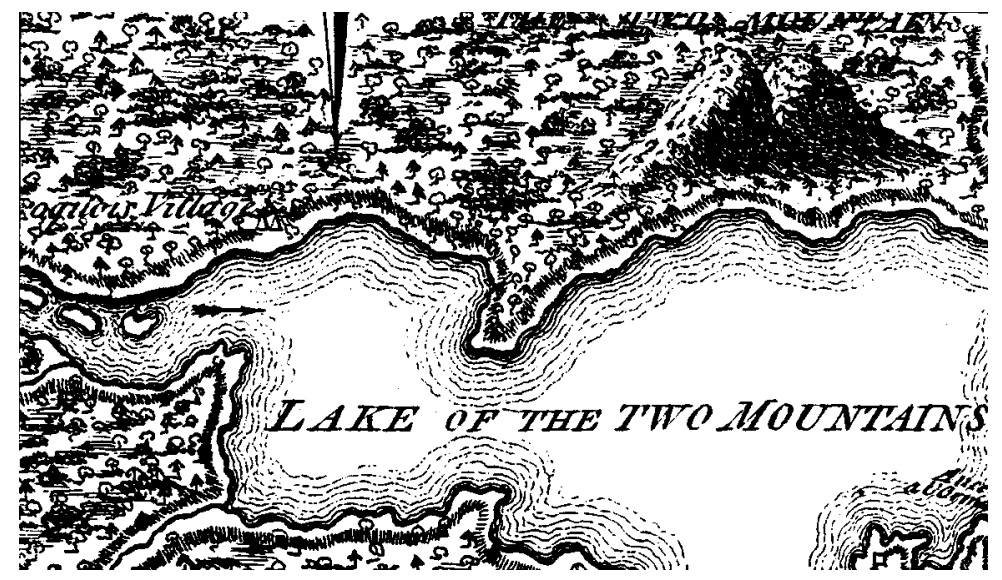

Partie de la carte The Isles of Montreal, as they have been survey'd by the French Engineers, 1761.

FIGURE 8

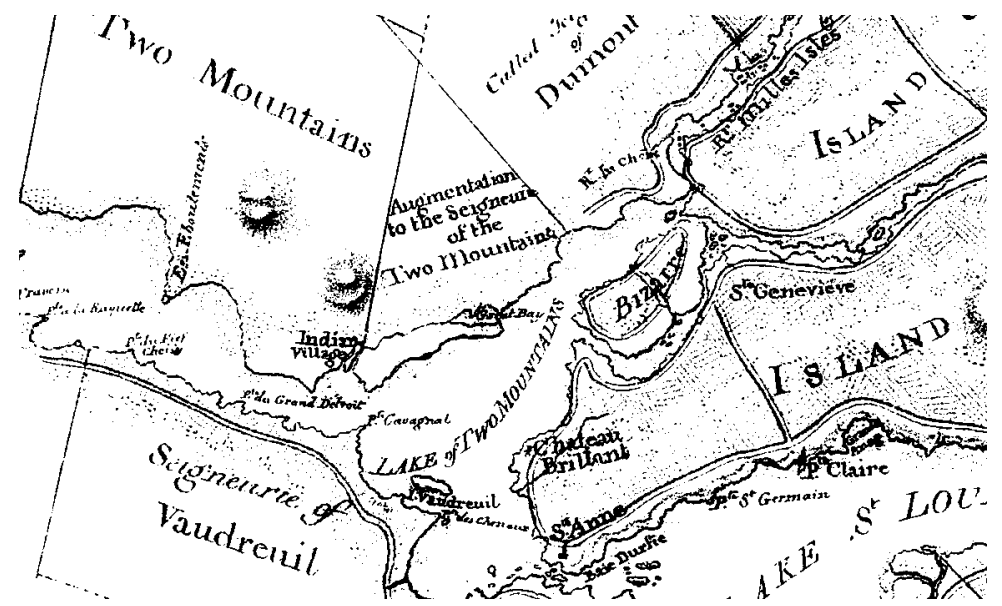

Partie du Plan of part of the province of Lower Canada... by S. Gale and J. B. Duberger, 1795. 
en partie visible que du haut de la montagne de Rigaud ou d'une des collines d'Oka et il serait peu vraisemblable que la vue de plusieurs sommets d'un côté du lac et d'un seul de l'autre côté ait conduit un observateur à conclure qu'il n'y avait que deux collines ou montagnes.

Il est difficile d'expliquer pourquoi la tradition orale rapporte que les deux montagnes sont le Calvaire et la montagne de Rigaud, car ces données vont à l'encontre de la logique qui découle de l'observation des lieux. Le fait que, jusqu'à une période relativement récente, seules les collines de Rigaud et du Calvaire avaient un nom y est peut-être pour quelque chose?

La montagne du Calvaire porte ce nom depuis 1749, année de la construction des chapelles sur son flanc ouest et à son sommet et il est vraisemblable que l'usage du nom de Rigaud pour désigner la montagne remonte au début du XIX ${ }^{\mathrm{e}}$ siècle. La notoriété de cette dernière se serait répandue parallèlement à celle du village du même nom. Elle n'est représentée sur aucune carte avant celle de Bouchette qui fut le premier à l'indiquer (sans la nommer) sur sa carte de 1815 .

Quant aux deux auteurs qui s'intéressent à l'histoire d'Oka, ils proposent les localisations suivantes: «[...] on attribua définitivement au lac, le nom de Lac des Deux-Montagnes à cause de la montagne du Calvaire et l'autre, celle nommée "Saint-Sulpice" au pied de laquelle se trouve le monastère des Trappistes. On a aussi pu lire que le lac des Deux-Montagnes devait son nom à la montagne du Calvaire d'Oka et à celle de Rigaud. D'autres pensaient que le lac des Deux-Montagnes devait son appellation à la colline du Calvaire et à celle du Mont-Bleu. Retenons que la plus plausible appellation est la première ${ }^{41}$.» Le deuxième «[...] affirme que ces deux montagnes sont toutes situées sur le même bord du lac, sur le côté nord, autrement dit en arrière d'Oka, que l'une est bien celle où a été édifié le Calvaire avec en arrière-plan d'autres collines, et l'autre, celle nommée "SaintSulpice", au pied de laquelle se trouve le Collège des Trappistes [...], ce sont les deux seuls "monts" que l'on voit lorsqu'on débouche sur le lac des Deux-Montagnes par les rapides au nord de l'île Perrot (Rapides de Brussi), $[\ldots]^{42} \gg$. Ce dernier a joint à son texte un dessin qui montre deux collines d'élévation et de forme assez semblables.

Le toponyme Saint-Sulpice désignant la colline au pied de laquelle se trouve le monastère des Trappistes est une appellation dont l'usage n'est pas très répandu et tout porte à croire que cette petite élévation fut ainsi baptisée par ces derniers ${ }^{43}$. En effet, c'est au sommet de cette colline qu'ils construisirent leur premier monastère en 1881, lequel devenu trop petit fut reconstruit au bas de cette même colline, mais du côté nord. Quelques années plus tard, ils érigèrent au

41. Stella Dupuis-Mailhot, op. cit., 1.

42. André De Pagès, loc. cit., 23.

43. «[...] le Père Jean-Baptiste Gaudin qui, [...] demeura à la maisonnette du meunier afin de diriger les travaux du premier monastère construit sur le haut de la colline Saint-Sulpice, comme nous l'avons dit plus haut.» C.-A. Doucet, La Trappe d'Oka (Montréal, 1979), 36, note 21. 


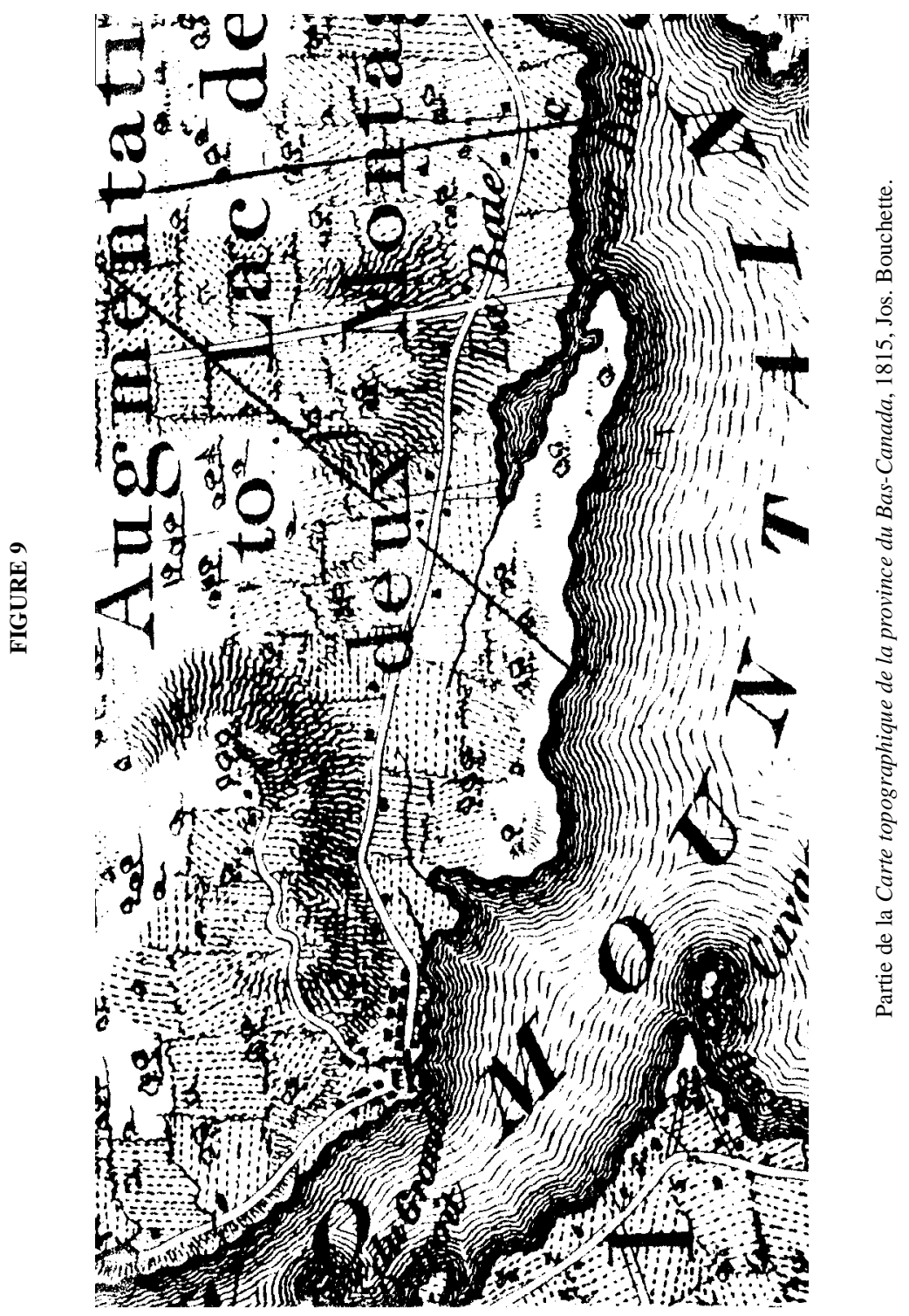


sommet de cette colline (Saint-Sulpice) un édifice en pierre qui abrita les étudiants de l'Institut agricole d'Oka jusqu'en $1962^{44}$.

Le dessin plutôt sommaire qui accompagne ce dernier texte pourrait être interprété comme une représentation de la montagne du Calvaire et de la montagne «Jumelle» au nord-est, mais son texte indique qu'il s'agit de la montagne du Calvaire et du mont Saint-Sulpice. Un examen attentif de la carte topographique aurait certainement convaincu cet auteur que le petit mont Saint-Sulpice (122 mètres) sis entre la montagne du Calvaire (190 mètres) et la montagne «Jumelle» au nord-est (220 mètres) ne pouvait être l'une des deux montagnes à l'origine du nom du lac. Quant aux deux autres localisations du premier auteur, elles ont déjà fait l'objet de commentaires.

\section{CONCLUSION}

Les documents disponibles nous indiquent que le toponyme Deux Montagnes fut en usage avant l'année 1674, mais ils ne permettent pas d'affirmer que le lac avait un nom avant l'arrivée des Blancs. Il n'est pas non plus possible de dire à qui, des Blancs ou des Indiens, on doit attribuer la paternité de cette appellation.

Quant à la situation des deux montagnes, les observations ont montré qu'à l'exception des trois ou quatre bosses de faible envergure au nord-ouest et au nord de la Grande Baie, quatre montagnes seulement sont clairement visibles du lac: la montagne du Calvaire, sa «Jumelle», la montagne de Rigaud et le mont Bleu. L'aspect physique des lieux permet donc deux endroits différents pour situer les deux montagnes: au nord-est d'Oka ou, dans la partie ouest du lac, la montagne de Rigaud et le mont Bleu.

Les cartes anciennes fournissent un argument important en faveur des deux montagnes au nord-est d'Oka. Toutes placent les deux montagnes du côté nord du lac et, de toutes celles qui ont été consultées, une seule, la plus récente, celle de 1815 de Bouchette, indique la montagne de Rigaud, les 13 autres n'en font pas mention. Cette omission réduit à néant la possibilité que la montagne de Rigaud soit l'une des deux montagnes et que ces dernières soient le couple montagne de Rigaud et mont Bleu.

De plus, même si l'on ne tient pas compte des trois premières cartes de Bellin, deux cartes seulement ${ }^{45}$, publiées assez tardivement d'ailleurs (1795 et 1813), sur les onze autres de la liste indiquent que les deux montagnes sont la montagne du Calvaire et le mont Bleu. Les neuf autres placent les deux symboles de montagne au nord-est d'Oka, vis-à-vis du bout de l'île de Montréal et l'entrée dans le lac par le passage entre cette dernière île et l'île Perrot.

44. «Plus tard en 1914, Dom Pacôme y construira le "Pensionnat", puis en 1930, il y ajoutera l'édifice en pierre que nous voyons encore sur la côte St-Sulpice.» Oka, ouvriers de la parole, 1881 1981. Abbaye Notre-Dame-du-Lac (Montréal, 1981), 20.

45. Voir les cartes 10 et 11 de la Liste des cartes. 
À l'avantage des deux montagnes au nord-est d'Oka, il faut aussi mentionner que leur élévation et leur forme arrondie très semblables sont remarquables dans le paysage, caractéristiques qu'avait notées le géographe Raoul Blanchard qui, le premier, les a qualifiées de jumelles. Ces traits spécifiques sont facilement observables lorsqu'on entre dans le lac par le passage plus haut mentionné.

Enfin, le dicton populaire à l'effet que, lorsqu'on se déplace sur le lac, l'une des montagnes cache l'autre, n'est vrai que pour la montagne du Calvaire et la montagne «Jumelle». Il ne peut absolument pas s'appliquer à la montagne de Rigaud et au mont Bleu situés de part et d'autre du lac, et encore moins à la montagne du Calvaire et au mont Bleu. Pour toutes ces raisons, il semble bien que les deux montagnes à l'origine du nom du lac soient la montagne du Calvaire et la montagne «Jumelle».

\section{ANNEXE 1}

\section{LISTE DES CARTES}

Cartes antérieures à 1816 sur lesquelles sont représentées au moyen de symboles au moins deux des montagnes suivantes: la montagne du Calvaire, la montagne «Jumelle», le mont Bleu et la montagne de Rigaud. Ces cartes ont été consultées à la Bibliothèque nationale du Québec.

1 - Carte de la partie orientale de la Nouvelle-France ou du Canada par N. Bellin (1744). Tiré de Histoire et description générale de la Nouvelle-France par le P. De Charlevoix.

2 - Partie orientale de la Nouvelle France ou du Canada par N. Bellin, 1745.

3 - Suite du cours du fleuve de St-Laurent, depuis Québec jusqu'au lac Ontario (1757) par N. Bellin. Cette carte fait partie de l'Atlas de l'hist[toire] g[énérale] des voya [ges] de [Antoine François Prévost, dit Prévost d'Exiles]-[Paris]: [Didot], [1780?]. 1 atlas (76 f.): 75 cartes; $27 \mathrm{~cm}$.

4 - Suite du cours du fleuve de St-Laurent depuis Québec jusqu'au lac Ontario par N. Bellin. [1757?].

5 - Partie du fleuve Saint-Laurent depuis Québec jusqu'au lac St-François (1764). Cette carte et la suivante sont tirées du Petit atlas maritime: recueil de cartes et plans des quatre parties du monde. Premier volume contenant l'Amérique septentrionale et les isles Antilles. [Bellin]. - [Paris: l'auteur, 1764]. Carte 10.

6 - L'Isle de Montréal et ses environs. Plan de la ville de Montréal ou Ville Marie. Tiré du même ouvrage que la carte précédente. Carte 11.

7 - Le fleuve Saint-Laurent, représenté plus en détail que dans l'étendue de la carte, [d'Anville]. 1755; carton de la carte: Canada, Louisiane, terres anglaises $[\ldots]$. 
8 - The isles of Montreal, as they have been survey'd by the French Engineers. -[London: London Magazine], ca 1761.

9 - A new map of the province of Quebec according to the royal proclamation of the 7th of october 1763 from the french surveys connected with those made after the war by captain Carver and other officers, in his majesty's service, 1776.

10 - Plan of the river LaChinay or Milles Isles with the lake of two mountains and part of Ottawa river [...] by Jos. Peachy D.P., 1793.

11 - A new and correct map of the province of Québec with the adjacent states and provinces [...] by captain Carver and other officers. - London: Laurie and Whittle, 1794.

12 - Plan of part of the province of Lower Canada [...], compiled [...] by Messrs Samuel Gale and John B. Duberger, 1795.

13 - A new map of the province of Lower Canada [... ] by Samuel Holland. - London: Willm. Faden, 1813.

14 - Carte topographique de la province du Bas-Canada par Joseph Bouchette. - Montréal, Éditions Élysées, 1980. Fac-similé de l'édition originale: to his royal highness George Augustus Frederick [...] this topographical map of the province of Lower Canada [...] - London: Faden, 1815. 